\title{
Distal Sensory Neuropathy and Pain in HIV-1 Patients: Retrospective Study
}

Babak A Rezaei ${ }^{1}$, Ruma Mukerjee ${ }^{2}$, Charles Groomes ${ }^{1}$, Varun Sethi ${ }^{1}$, Tinatin Chabrashvili ${ }^{1}$ and Bassel E Sawaya ${ }^{1,2 *}$

${ }^{1}$ Department of Neurology, Molecular Studies of Neurodegenerative Diseases Lab, USA

${ }^{2}$ The FELS Institute for Cancer Research, Temple University School of Medicine, Philadelphia, USA

\begin{abstract}
HIV-1 associated neuropathy remains an issue that interferes with patient's quality of life. Distal Sensory Peripheral Neuropathy (DSPN) is the common neuropathy associated with HIV-1 infection. We studied the role of HIV-1 in developing DSPN in 157 HIV-positive patients. Our results pointed to the potential role of HIV-1 in the etiology of DSPN and pain syndrome.
\end{abstract}

Keywords: HIV-1; Sensory Neuropathy; Pain

\section{Introduction}

It is well recognized that patients infected with HIV-1 suffer from different forms of pain in addition to other neurological symptoms [1]. In addition, peripheral neuropathy is the most frequent neurological complication in HIV-1 infected individuals. Unfortunately, these symptoms persist even with the use of the combination of antiretroviral therapy (cART) [2]. Among neuropathies, distal sensory peripheral neuropathy (DSPN) is the most common neuropathy associated with HIV-1 infection [3]. DSPN frequently manifests as a sensory neuropathy, and associated pain syndrome has been acknowledged. Several factors have been implicated in development of HIV-associated neuropathy including the cART [4]. In addition, the pain can also arise directly because of bone disease such as osteopenia/osteoporosis that could be associated with HIV-1 infection [5]. Interestingly, an HIV-1 patient might not be aware that some nerves are damaged because of the infection. In this regard, a study published in 2009 demonstrated that a significant number of these patients suffered from a mild pain [6]. Further, it has been suggested that HIV-1 associated neuropathy can be the side effect of medications used by HIV-1 patients. As a result, these medications damage the nerves especially the mitochondria and cause certain type of pain [6,7]. Therefore, it was suggested that clinicians should consider and weigh the risks and benefits of each drug before initiating any HIV-related neuropathy treatment [8]. Furthermore, it has been shown that an increase in pain especially DSPN was observed in HIV-infected patients who used or using drugs of abuse [9]. However, the exact mechanisms leading to this pain remain to be identified. In this retrospective study, we examined the incidence of DSPN and its relationship with pain syndrome in 157 HIV-positive patients that were seen at Temple University Medical Center neurology clinic between 2007 and 2012. Our data corroborate with the published data and confirmed our hypothesis regarding the potential role of HIV-1 infection in the etiology of DSPN and pain syndrome, and further emphasis the necessity of research to identify the mechanisms involved.

\section{Methods}

\section{Patients}

157 patients older than 18 years of age infected with HIV-1, who visited the neurology clinic (mainly as outpatients) over five year period between 2007 and 2012 .

\section{Inclusions criteria}

Inclusion criteria were: I. HIV-positive patients seen in Neurology clinic. II. Sensory symptoms for at least 3 months including numbness, tingling (pins and needles sensation) and pain. III. EMG/NCS (Electromyogram / Nerve Conduction Studies) positive findings for any sensory neuropathy (DSPN, mononeuritis, autonomic neuropathies and radiculopathy). IV. Documentation regarding presence or absence of sensory symptoms and pain.

\section{Exclusion criteria}

Exclusion criteria were: I. Acute pain of somatic origin; II. Sensory symptoms started before diagnosis of HIV-1 infection; III. No documentations regarding their sensory symptoms. All patients had a complete neurological examination.

\section{Disclosure}

Identities of the patients were not revealed as well as all personal information.

\section{Results}

We were able to identify 157 patients infected with HIV-1 who were seen in our clinic as out or inpatients between the periods extending from January 1st 2007 through December 2012.

First, we sought to examine the percentage of HIV-1 infected patients that developed neuropathy. As shown in Figure 1A, HIV-1-infected patients were divided into two groups. 117 patients (G1) out of 157 patients with diagnosis of HIV-1 infection did not show symptoms of distal sensory neuropathy. However, distal sensory neuropathy was prevalent in 40 (25.4\%) patients (G2) of the 157 sampled patients infected with HIV-1.

*Corresponding author: Bassel E Sawaya, Molecular Studies of Neurodegenerative Diseases Lab Department of Neurology and The FELS Institute for Cancer Research Temple University School of Medicine3307 North Broad Street, MRB \# 719 Philadelphia, Pennsylvania 19140, USA, Tel: 215-707-5446; Fax: 215-707-5948; E-mail: sawaya@temple.edu

Received May 07, 2014; Accepted July 07, 2015; Published July 18, 2015

Citation: Rezaei BA, Mukerjee R, Groomes C, Sethi V, Chabrashvili T, et al. (2015) Distal Sensory Neuropathy and Pain in HIV-1 Patients: Retrospective Study . J AIDS Clin Res 6: 485. doi:10.4172/2155-6113.1000485

Copyright: $\odot 2015$ Rezaei BA, et al. This is an open-access article distributed unde the terms of the Creative Commons Attribution License, which permits unrestricted use, distribution, and reproduction in any medium, provided the original author and source are credited. 
To further evaluate the relationship between the pain and sensory neuropathy associated with HIV-1 infection, we then examined the presence of pain that patients from G2 experienced. As shown in Figure 1B, 25 out of 40 patients (a [62.5\%]) suffered from distal sensory neuropathy associated with pain, while 10 patients (b [25\%]) had non-painful distal sensory neuropathy confirmed by EMG/NCS, and 5 patients (c [12.5\%]) had clinically documented sensory symptoms without any pain or EMG/NCS finding.

Next, we sought to examine the percentage of comorbidities in patients with sensory neuropathy. As shown in Figure 2A, no signs of comorbidities were found in $11(27.5 \%)$ of these patients (G2). However, the remaining $29(72.5 \%)$ patients suffered from one or more type of comorbidities (Figure 2A).

Finally, we evaluated the type of comorbidities existing in the 29 patients. As shown in Figure 2B, all the comorbidities among these 29 patients could be categorized in four different subgroups: 12 patients with psychiatric problems (depression, anxiety), 6 patients suffered from diabetes, 8 patients had degenerative disc disease or carpal tunnel syndrome and 10 patients had prior history of ischemic stroke or Migraine. Note that the some patients were diagnosed with more than one symptom, which explains the number obtained. This observation corroborate with previous studies where neurological complications have been determined as an important cause of morbidity in the HIV/ AIDS population [10].

Nevertheless, to determine the percentage of patients suffering
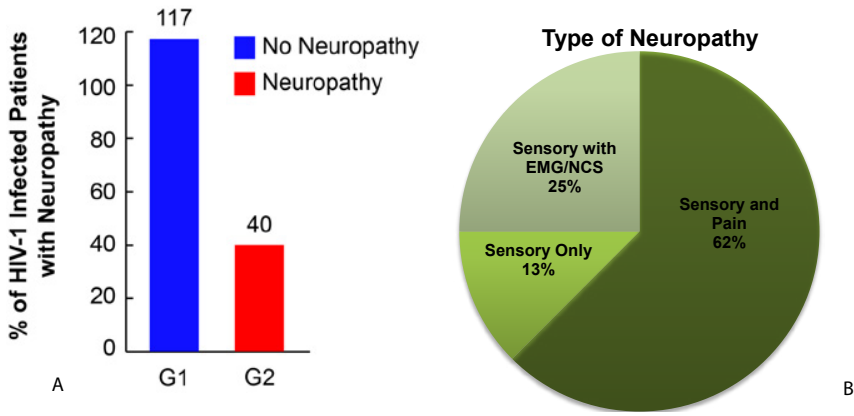

Figure 1: . Percentage of HIV-1 infected patients suffering from distal sensory neuropathy. A. $157 \mathrm{HIV}-1$ infected patients were admitted in our clinic between 2007 and 2012. The patients were divided into two groups. Only 40 patients (25.5\%) (G2) suffered from DSNP. B. Patients from G2 were divided into three subgroups a- sensory neuropathy associated with pain $(62.5 \%)$; b- sensory neuropathy without neuropathic pain; and c- $12.5 \%$ of patients had sensory symptoms without any pain or EMG/NCS findings. The results are statistically significant using Student's t test $(p<0.05$ compared with the Mock control group [one-way analysis of variance and ANOVA Test]).
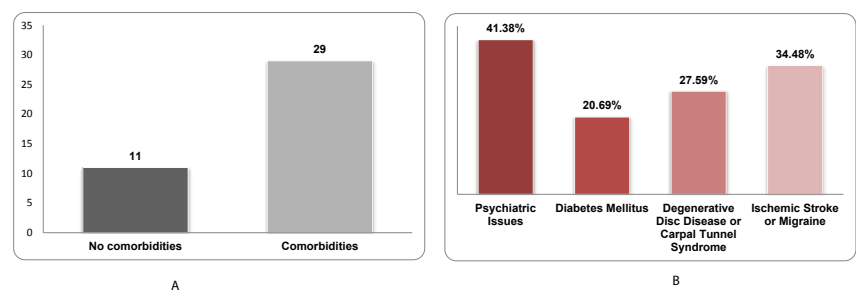

Figure 2: Percentage of patients with Comorbidities. A. Histogram displays the percentage of HIV-1 infected patients selected from G2 and show signs of comorbidities (29 out of 40 patients). B. Types of comorbidity observed in 29 patients. Note that some patients were diagnosed with more than one type of comorbidity. from neuropathy independently of neurological complications and diabetes, we excluded those suffering from ischemic stroke, Migraine, degenerative disc disease or carpal tunnel (18 patients) and/or from diabetes (6 patients). As a result, only 12 patients (52\%) with psychiatric problems will remain when compared to patients with no comorbidities (48\%).

\section{Discussion}

HIV-1 associated neuropathy is among the most frequent neurological complications associated with HIV-1 infection. This phenomenon remains prevalent even in the presence of cART. In this study, we examined the files of 157 HIV-infected patients evaluated in our clinic between 2007 and 2012 and suffering from pain by focusing only on sensory neuropathy. Our data confirmed our hypothesis that DSPN is associated with HIV-1 infection. We also examined the pain and the percentage of comorbidities involved. This demographic review of HIV-1 sensory neuropathy and pain corroborate with the national data (\%) and can possibly contribute to the decision making for future treatment.

Although comorbidities were inseparable part of causing sensory neuropathy in HIV patients but our results underlined the importance of HIV as an independent risk factor for distal sensory polyneuropathy and showed specifically neuropathic pain in about third of our patient population without any comorbidities. Our study is not without precedent, in this regard, it has been shown that Neurological complications, particularly central nervous diseases, are an important cause of morbidity in the HIV/AIDS population [10,11]. In both published studies, percentage of patients infected with HIV-1 and suffering from stroke that led to neuropathy was almost similar to our numbers. Thus, we concluded that ischemic stroke is an important cause of neuropathy depending on the anatomical area affected.

Given the nature of our study design, inevitably there were some limitations including poor documentations, selection bias and individual variability. Hence, dedicated randomized trials to investigate sensory neuropathy and specifically pain in HIV patients are warranted.

Overall, HIV- associated neuropathy can be divided and classified into several categories based on their pathology (axonal or demyelinating), time of appearance during HIV-infection and whether they are associated with opportunistic infection such as CMV [12]. Previous data suggests that DSPN is prevalent in $9 \%$ to $63 \%$ of the HIV population [13-18]. Some of the factors used to explain this broad spectrum of variability include differences i) in the degree of immunosuppression, ii) in the definition of neuropathy and iii) in the exposure to neurotoxins such as antivirals [19-21]. For fact, data from an earlier study suggests that viral suppression of HIV-1 through immune restoration may decrease the risk of developing DSPN [14]. Another study showed that pain was one of the most common initial symptom of neuropathy associated with HIV-1, with pain in the soles being the most common location [16]. In another study, it was shown that the risk of general neuropathy was associated with exposure to neurotoxins such as nucleoside analogs. Therefore, Clinicians should consider and weigh the risks and benefits of each drug before initiating any HIV-related neuropathy treatment [8].

Unfortunately, the pathogenesis of DSPN is incompletely understood and thought to be multifactorial. Its pattern of distal length dependent neuropathy, known as "dying back" neuropathy, is not unique and often indistinguishable from other toxic neuropathies [21]. Furthermore, it is not understood how the virus mediates inflammation 
and neuronal damage. Almost all the recovered virus from peripheral nerves was found inside macrophages and monocytes [21-25]. Two mechanisms of HIV-1 mediated neuronal injury are proposed and involved apoptosis [26] however, it is also entirely possible this neuronal injury is the result of collateral damage by the immune system, upon activation by the viral antigens.

In addition to the debate about mechanism, in the HIV-1 population there are several other risk factors for neuropathy that may serve as confounders. Namely, these are psychiatric illnesses, diabetes mellitus, degenerative disc disease, carpal tunnel syndrome, ischemic stroke, and migraines. In our study, we looked at the role that HIV-1 may play as an independent risk factor in developing distal sensory polyneuropathy. Taken together, existing observations demonstrated the need of a mechanistic study to identify the cellular and molecular mechanisms involved in the development of HIV-associated neuropathy. We believe that validating these results using animal model and developing new treatment for HIV-1 induced sensory neuropathy and pain could overturn this major complication and health care problem in our society.

\section{References}

1. Kranick SM, Nath A (2012) Neurologic complications of HIV-1 infection and its treatment in the era of antiretroviral therapy. Continuum ,[Minneap Minn] 18: 1319-1337.

2. Evans SR, Lee AJ, Ellis RJ, Chen H, Wu K, et al. (2012) HIV peripheral neuropathy progression: protection with glucose-lowering drugs? J Neurovirol 18: $428-433$

3. Ellis RJ, Marquie-Beck J, Delaney P, Alexander T, Clifford DB, et al. (2008) Human immunodeficiency virus protease inhibitors and risk for peripheral neuropathy. Ann Neurol 64: 566-572.

4. Erlandson KM, Allshouse AA, Jankowski CM, Duong S, MaWhinney S, et al. (2012) Risk factors for falls in HIV-infected persons. J Acquir Immune Defic Syndr 61: 484-489.

5. Jhaveri MA, Mawad HW, Thornton AC, Mullen NW, Greenberg RN (2010) Tenofovir-associated severe bone pain: I cannot walk! J Int Assoc Physicians AIDS Care 9: 328-334.

6. Valcour V, Yeh TM, Bartt R, Clifford D, Gerschenson M, et al. (2009) AIDS Clinical Trials Group (ACTG) 5157 protocol team: Acetyl-I-carnitine and nucleoside reverse transcriptase inhibitor-associated neuropathy in HIV infection. HIV Med 10: 103-110.

7. Holzinger ER, Hulgan T, Ellis RJ, Samuels DC, Ritchie MD, et al. (2012) Mitochondrial DNA variation and HIV-associated sensory neuropathy in CHARTER. J Neurovirol 18: 511-520.

8. Smith HS (2011) Treatment considerations in painful HIV-related neuropathy. Pain Physician 14: E505-24.

9. Robinson-Papp J, Gelman BB, Grant I, Singer E, Gensler G, et al. (2012) National NeuroAIDS Tissue Consortium: Substance abuse increases the risk of neuropathy in an HIV-infected cohort. Muscle Nerve 45: 471-476.

10. Isezuo SA, Sani AZ, Ezunu E, Maiyaki S, Njoku CH, et al. (2009) Clinical neuropathy in HIVIAIDS: an eight-year review of hospitalized patients in Sokoto, northwestern Nigeria. Trop Doct 39: 133-135.

11. Jowi JO, Mativo PM, Musoke SS (2007) Clinical and laboratory characteristics of hospitalised patients with neurological manifestations of HIVIAIDS at the Nairobi hospital. East Afr Med J 84: 67-76.

12. Kolson DL, Gonzalez-Scarano F (2001) HIV-associated neuropathies: role of HIV-1, CMV, and other viruses. J Peripher Nerv Syst 6: 2-7.
13. Cherry CL, Skolasky RL, Lal L, Creighton J, Hauer P, et al. (2006) Antiretroviral use and other risks for HIV-associated neuropathies in an international cohort. Neurology 66: 867-873.

14. Lichtenstein KA, Armon C, Baron A, Moorman AC, Wood KC, et al. (2005) HIV Outpatient Study Investigators: Modification of the incidence of drugassociated symmetrical peripheral neuropathy by host and disease factors in the HIV outpatient study cohort. Clin Infect Dis 40: 148-157.

15. Maschke M, Kastrup O, Esser S, Ross B, Hengge U, et al. (2000) Incidence and prevalence of neurological disorders associated with HIV since the introduction of highly active antiretroviral therapy (HAART). J Neurol Neurosurg Psychiatry 69: 376-380.

16. Schifitto G, McDermott MP, McArthur JC, Marder K, Sacktor N, et al. (2002) Dana Consortium on the Therapy of HIV Dementia and Related Cognitive Disorders: Incidence of and risk factors for HIV-associated distal sensory polyneuropathy. Neurology 58: 1764-1768.

17. Simpson DM, Kitch D, Evans SR, McArthur JC, Asmuth DM, et al. (2006) ACTG A5117 Study Group: HIV neuropathy natural history cohort study: assessment measures and risk factors. Neurology 66: 1679-1687.

18. So YT, Holtzman DM, Abrams DI, Olney RK (1988) Peripheral neuropathy associated with acquired immunodeficiency syndrome. Prevalence and clinical features from a population-based survey. Arch Neurol 45: 945-948.

19. Barohn RJ, Gronseth GS, LeForce BR, McVey AL, McGuire SA, et al. (1993) Peripheral nervous system involvement in a large cohort of human immunodeficiency virus-infected individuals. Arch Neurol 50:167-171.

20. Simpson DM, Katzenstein DA, Hughes MD, Hammer SM, Williamson DL, et al. (1998) Neuromuscular function in HIV infection: analysis of a placebocontrolled combination antiretroviral trial. AIDS Clinical Group 175/801 Study Team. AIDS 12: 2425-2432.

21. Brinley FJ Jr, Pardo CA, Verma A (2001) Human immunodeficiency virus and the peripheral nervous system workshop. Arch Neurol 58:1561-1566.

22. Chaunu MP, Ratinahirana H, Raphael M, Hénin D, Leport C, et al. (1989) The spectrum of changes on 20 nerve biopsies in patients with HIV infection. Muscle Nerve 12: 452-459.

23. De la Monte SM, Gabuzda DH, Ho DD, Brown RH Jr, Hedley-Whyte ET, et al. (1988) Peripheral neuropathy in the acquired immunodeficiency syndrome. Ann Neurol 23: 485-492.

24. Ho DD, Rota TR, Schooley RT, Kaplan JC, Allan JD, et al. (1985) Isolation of HTLV-III from cerebrospinal fluid and neural tissues of patients with neurologic syndromes related to the acquired immunodeficiency syndrome. N Engl J Med 313: 1493-1497.

25. Rizzuto N, Cavallaro T, Monaco S, Morbin M, Bonetti B, et al. (1995) Role of HIV in the pathogenesis of distal symmetrical peripheral neuropathy. Acta Neuropathol 90: 244-250.

26. Melli G, Keswani SC, Fischer A, Chen W, Hoke A (2006) Spatially distinct and functionally independent mechanisms of axonal degeneration in a model of HIV-associated sensory neuropathy. Brain 129:1330-1338. 